\title{
Simulation of kinetic envelope systems and their relation to increased thermal comfort - adaptive model in interior spaces Case of the Caribbean Region, Colombia
}

XXIV International Conference of the Iberoamerican Society

\author{
John Sebastian Estupiñan \\ Universidad Nacional de Colombia | Colombia | jsestupinana@unal.edu.co \\ Rodrigo Shiordia López \\ Universidad Anáhuac México | México | rodrigo.shiordia@anahuac.mx \\ Andrés Felipe Pérez \\ Universidad Nacional de Colombia | Colombia | afperezm@unal.edu.co \\ Rodrigo Velasco \\ Colombia | rodrigo@frontis3d.co
}

\begin{abstract}
In Colombia the last five years the average annual demand for air conditioning increased by $73 \%$. One strategy to increase thermal comfort and reduce the use of cooling systems and energy consumption in buildings is the use of kinetic envelopes as an active response system to changes in the environment. However, there is little literature analyzing their performance and effects on buildings. This research analyses the operation of different envelope systems through digital modelling and simulation of environmental conditions. The aim is to generate a set of defined advantages in the use of kinetic envelopes for the context in the Caribbean region in Colombia.
\end{abstract}

Keywords: Kinetic envelopes; Digital simulation; Climatic context; Thermal comfort; Energy consumption.

\section{INTRODUCCIÓN}

El termino envolvente principalmente destaca y diferencia la función entre revestimiento y estructura de la edificación, sin embargo, actualmente se consolida la teoría de que dicho revestimiento corresponde a un sistema ambiental inteligente dado que actúa como interfaz entre el interior y exterior de la edificación, intercambiando energía e información proveniente del entorno (López et al., 2017).

En Colombia, según cifras del reporte "World Air Conditioner Demand by Región" para el periodo 2013 2018 se registró un aumento del $73 \%$ en la demanda promedio anual (Japan Refrigeration and Air Conditioning Industry Association, 2019); Lo anterior plantea un problema determinado por el incremento del consumo de energía proveniente del campo de la construcción, además a nivel regional es el país con mayor aumento porcentual, salvo Chile con un aumento del $50 \%$, a diferencia de Brasil, Argentina, México o Panamá que presentan consumos constantes o disminuciones con el paso de los años.

Este documento se centra en el enfoque que la tecnología en envolventes plantea, tales como los principios cinéticos y adaptables alcanzados mediante alternativas en modelado y simulación digital, así como la preocupación constante en la reducción de impactos en el consumo de energía en las edificaciones (Velasco et al., 2015). Lo anterior, con el objetivo de reconocer ventajas del uso de envolventes cinéticas $(E C)$ mediante la posibilidad de identificar una serie de condiciones características y principios de diseño en un contexto como la región caribe de Colombia, con la intención de aumentar el confort térmico (CTMA) y reducir el uso de sistemas de enfriamiento que a su vez reduzca el consumo de energía.

Rhino/Grasshopper - LadyBug/Honeybee son software utilizados en el desarrollo del estudio que tienen cualidades y beneficios desde la perspectiva de la vinculación entre diferentes actividades, donde el modelado, parametrización, análisis y visualización de resultados ocurren de forma simultánea (Mackey \& Roudsari, 2017), lo cual facilita la extracción de resultados en cada uno de los procesos realizados.

La investigación desarrollada lleva a cabo un grupo de simulaciones de condiciones de confort térmico (CTMA) para un grupo experimental de cuatro envolventes cinéticas $(E C)$ y además para un grupo de control, con una envolvente sin elementos de protección solar y otra de elementos tipo persiana; las cuatro envolventes dentro del grupo experimental son definidas con anterioridad mediante un proceso de selección que hace parte de un estudio más extenso.

\section{CONFIGURACIÓN DEL CONTEXTO}

La simulación utiliza tres grupos de insumos para construir el contexto de análisis: estrategias digitales, contexto climático y contexto edificado o espacio de análisis.

\section{HERRAMIENTAS DIGITALES RHINO 3D,} GRASSHOPPER / LADYBUG, HONEYBEE

En este estudio Rhinoceros cumple la función de ser el software base de visualización y creación de geometrías básicas, un ejemplo de estas es la necesaria para definir 
espacios de simulación y de elementos interiores; en esta herramienta no se modelan ni crean superficies complejas, se utilizan pluggins y complementos que Rhinoceros (Robert McNeel \& Associates, 2013) ofrece como es el caso de Grasshopper.

Uno de los componentes soportados por Grasshopper es LadyBug Tools, una colección de aplicaciones informáticas gratuitas enfocadas en la educación y el diseño ambiental que en términos sencillos cumplen la función del análisis relacionado con datos climáticos y meteorológicos, HoneyBee dentro de esta colección de aplicaciones es la herramienta de mayor importancia para la realización de simulaciones en la investigación. Tiene la función de desarrollar ejercicios de iluminación detallada y modelos termo dinámicos en espacios de simulación, que para etapas de diseño y posteriores tiene gran importancia, esta herramienta se ejecuta mediante varios grupos de motores, en este caso se generan flujos de temperatura que usan motores como Berkeley Lab Therm / Window. El enfoque especializado de HoneyBee para simulaciones de confort la convierten en una de las herramientas más completas desarrolladas en la actualidad en el campo del análisis ambiental (Mostapha Sadeghipour Roudsari, 2013).

El análisis llevado a cabo en este documento evalúa condiciones ambientales internas, principalmente condiciones de temperaturas y CTMA para geometrías de EC utilizando el paquete de software descrito anteriormente.

\section{ANÁLISIS DE CONEXTO CLIMATICO BARRANQUILLA, COLOMBIA}

Barranquilla es capital del departamento del Atlántico, clasificada dentro de la escala de climática köppen como Aw - Tropical de sabana con invierno seco (Kottek et al., 2006). La temperatura de la ciudad a lo largo del año tiene promedios mensuales entre los 27 y $28^{\circ} \mathrm{C}$, con su máximo el mes de mayo de $28.5^{\circ} \mathrm{C}$ y con su pico mínimo el mes de enero con $27{ }^{\circ} \mathrm{C}$. La radiación anual promedio llega a $234 \mathrm{Wh} / \mathrm{m}^{2}$ con variaciones importantes durante el año, con pico máximo el mes de abril con $264 \mathrm{Wh} / \mathrm{m}^{2}$ y con su pico mínimo el mes de noviembre con $198 \mathrm{Wh} / \mathrm{m}^{2}$. La humedad relativa en la ciudad a lo largo del año no muestra variaciones importantes mes a mes, el promedio anual es de $82 \%$, donde octubre es el mes con mayor humedad con un $87 \%$ y con índice mínimo el mes de febrero con un promedio de $75 \%$.

Tabla 1: Indicé de promedio mensual de radiación en Barranquilla

\begin{tabular}{lcc}
\hline \multicolumn{1}{c}{ Mes } & Radiación $\left(\mathrm{Wh} / \mathrm{m}^{2}\right)$ & Temperatura $\left({ }^{\circ} \mathrm{C}\right)$ \\
\hline Enero & 217.16 & 27.05 \\
Febrero & 239.03 & 27.23 \\
Marzo & 257.32 & 27.48 \\
Abril & 264.67 & $\mathbf{2 8 . 1 3}$ \\
Mayo & 240.61 & 28.51 \\
Junio & 237.03 & 28.49 \\
Julio & 236.53 & 28.40 \\
Agosto & 247.62 & 28.36 \\
Septiembre & 234.58 & 28.14 \\
Octubre & 232.08 & 27.64 \\
Noviembre & 198.48 & 27.68 \\
Diciembre & 208.79 & 27.71 \\
\hline
\end{tabular}

La fecha de análisis seleccionada de acuerdo al análisis mensual de radiación es el mes de abril, mes con mayor promedio dentro del año y seguido a esto el día 18 correspondiente al mayor promedio de radiación con 284 $\mathrm{Wh} / \mathrm{m}^{2}$ dentro del mes, (Tabla 1); las horas de análisis definidas para los ejercicios de simulación de condiciones de confort térmico - modelo adaptativo serán cinco: 7:00 am, 10:00 am, 12:00 pm, 3:00 pm y 5:00 pm.
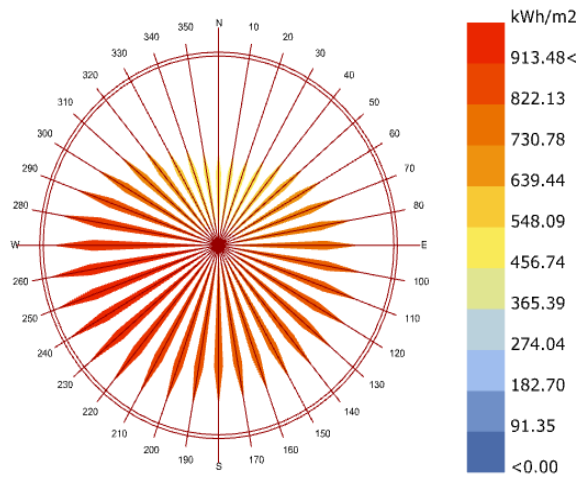

Total Radiation $(\mathrm{kWh} / \mathrm{m} 2)$

Barranquilla_Cortissoz_Intl_AP_ATL_COL_2006

1 JAN 6:00 - 31 DEC 18:00

Figura 1: Rosa de radiación anual promedio para la ciudad de Barranquilla.

También se debe determinar junto con los parámetros de contexto como se muestra en la Figura 1 la dirección en la cual hay mayor índice de radiación, es así como la gráfica permite concluir que la dirección con mayor incidencia de radiación anual promedio es la sur - occidente, en horas comprendidas entre las 6:00 am y las 6:00 pm (rango de horas de análisis).

Finalmente es importante definir los rangos de temperatura deseados, ya que como se ha mencionado anteriormente la etapa de simulación comprende estándares que, para el caso de condiciones de CTMA, se analizan a continuación:

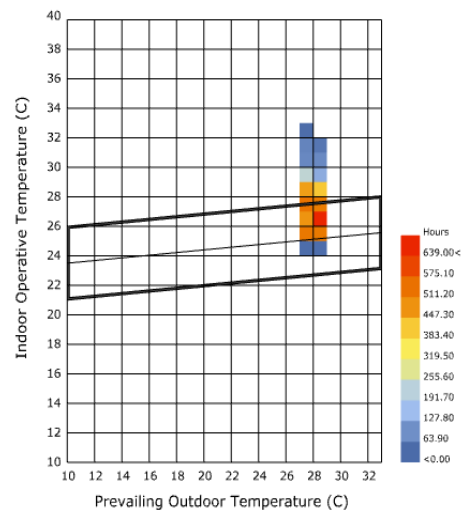

\section{Adaptive Chart \\ Barranquilla Cortissoz Intl AP_ATL COL} 1 JAN 7:00 - 31 DEC 18:00

Figura 2: Diagrama de CTMA en rangos de las horas evaluadas a lo largo del año para la ciudad de Barranquilla.

El diagrama observado en la Figura 2 se crea bajo estándares de la norma ANSI/ASHRAE Standard 55 
(ANSI/ASHRAE, 2017), para horas de ocupación comprendidas entre las 7:00 am y 6:00 pm, a lo largo del año con una temperatura promedio exterior de $27.9^{\circ} \mathrm{C}$, promedio suministrado por el documento epw (energy Plus Weather file, por sus siglas en inglés) de datos meteorológicos para Barranquilla, el diagrama tiene parámetros de entrada correspondientes a la temperatura media de aire interior, temperatura media radiante interior, velocidad de viento interior y temperatura media exterior, también se especifica la lectura de parámetros bajo la normativa indicada, sin embargo en este caso se cambia el parámetro de nivel de adaptabilidad de ventilación natural a ventilación artificial, pues los ocupantes tienden a presentar niveles de adaptación con sistemas de aire acondicionado.

\section{EDIFICACIÓN - ESPACIO DE ANÁLISIS BARRANQUILLA, COLOMBIA}

El edificio seleccionado dentro de la ciudad de Barranquilla es el Centro Empresarial Green Tower, un edificio categorizado como el sexto más alto de barranquilla, con aproximadamente $24000 \mathrm{~m}^{2}$ construidos y uso de oficinas, además su envolvente está compuesta en su totalidad por muros cortina y esta climatizado en cada uno de sus espacios. La unidad de oficina seleccionada para el ejercicio de simulación es la localizada en la orientación sur - occidente del edificio (Figura 3), debido al análisis de incidencia de radiación solar descrito en el análisis de contexto climático.
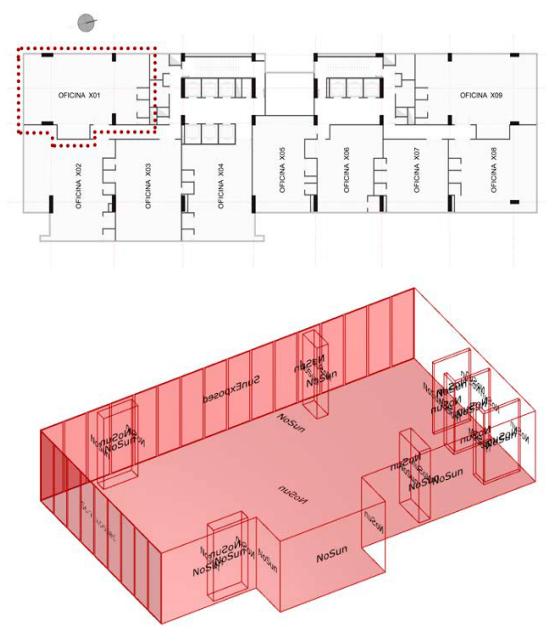

Figura 3: Planta general oficina X01, espacio de simulación Rhino / Grasshopper.

Como lo muestra la Figura 3 la oficina X01 señalada en la zona superior de la ilustración se ubica en la zona sur occidente del edificio, con exposición solar en dos planos de fachada; la oficina tiene un área construida de $149 \mathrm{~m}^{2} \mathrm{y}$ es una de las más grandes dentro de la planta tipo de oficinas, además dentro del espacio modelado para este ejercicio se agregan los elementos estructurales como columnas y también muros divisorios en zonas de servicio, que son caracterizados dentro de Honeybee como muros interiores sin exposición solar, condición que también se aplica para muros delimitadores, piso y techo, así se restringe la condición de exposición solar a los planos de fachada que también se caracterizan con una partición de acristalamiento similar a las condiciones reales de la edificación.

\section{CONCLUSIÓN}

Condiciones de contexto enmarcadas para Barranquilla, con fecha de análisis el 18 de abril, en cinco horas de evaluación a lo largo del día: 7:00 am, 10:00 am, 12:00 pm, 3:00 pm y 5:00 pm; además el resultado del análisis del grafico de confort térmico de modelo adaptativo indica una temperatura objetivo de $25.1{ }^{\circ} \mathrm{C}$ como temperatura de sensación de bienestar, también dentro del polígono de temperaturas aceptables se consideran $2{ }^{\circ} \mathrm{C}$ menores y superiores a la temperatura objetivo conformando un rango de temperatura aceptable entre 23.1 y $27.1^{\circ} \mathrm{C}$. E espacio de análisis seleccionado para el estudio es la oficina X01 ubicada al sur occidente de la planta tipo del edificio Centro Empresarial Green Tower.

\section{SIMULACIÓN DE CONFORT TERMICO - MODELO ADAPTATIVO (CTMA)}

Se parte con un grupo de cinco simulaciones, de estas hay tres de carácter térmico, que funcionan como insumo para dos simulaciones de CTMA, también se plantea un contexto experimental explicado con anterioridad con respecto a herramientas, clima y espacio de análisis, es por esto que este capítulo no ahondara en la construcción de contexto más que en la aclaración de algunos conceptos y definiciones de la metodología de simulación. El ejercicio contiene un grupo de seis casos que finalmente va a conformar un conjunto de once mil ochocientos cincuenta (11850) simulaciones, cinco por cada una de los seis casos, y a su vez por cinco adicionales correspondientes a las horas de análisis, lo anterior por la comprobación en cada posición de las envolventes que suman setenta y nueve de los seis casos.

En modelado de los casos, el detalle se define a un alcance de geometría principal del sistema, con replicación del movimiento y materialidad, el análisis plantea un patrón general de movimiento para los dos planos de envolvente, que de igual forma tiene un nivel de control Nivel 1 (Velasco et al., 2015).

\section{EJERCICIO DE SIMULACIÓN}

El algoritmo se estructura de forma que pueda cubrir un grupo de cinco simulaciones simultáneamente, estas corresponden a un primer conjunto de análisis de temperatura: a) temperatura radiante media, b) temperatura de aire y c) temperatura operativa, recordando que esta última es el resultado del promedio en la suma de la temperatura radiante media y la temperatura de aire; el segundo conjunto de análisis es el resultado de la visualización de índices de porcentaje de aceptación de temperatura y los índices de CTMA que calcula la media de temperatura aceptable y define rangos en grados por debajo o por encima de dicha media.

\section{CONFORT TERMICO MODELO ADAPTATIVO (CTMA):} la representación de esta variable es de especial importancia como resultado final de simulación, porque permite definir el grado de diferencia en temperatura que cada punto de lectura tiene con la temperatura operativa optima definida anteriormente, la simulación en este caso representa la temperatura operativa optima con $0{ }^{\circ} \mathrm{C}$ y 


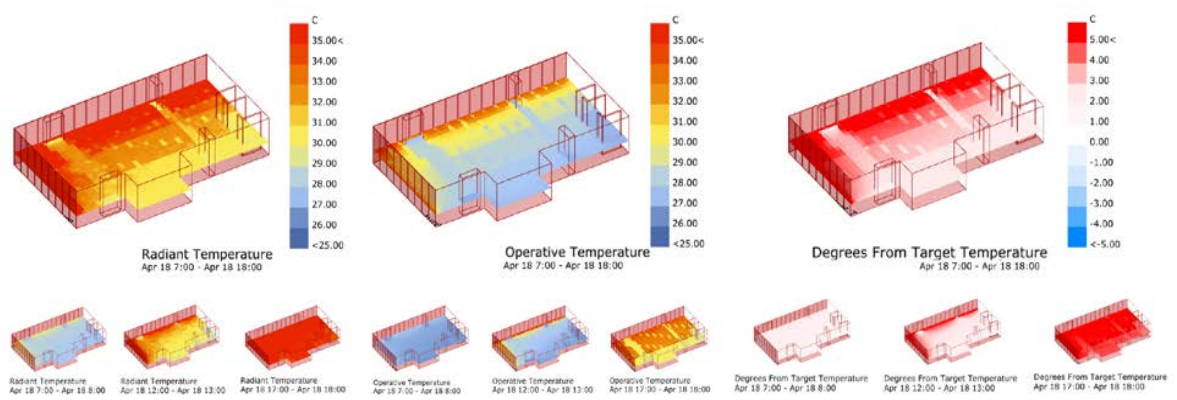

Figura 4: Simulación de Temperatura Radiante (tr), Temperatura Operativa (to), Confort Adaptativo (CTMA) en oficina tipo.

define rangos en -5 y $5^{\circ} \mathrm{C}$. Es importante recordar que la métrica aceptada mostrada por el diagrama de CTMA en el apartado análisis de contexto muestra temperaturas aceptables con diferencias de máximo hasta -2 o $2^{\circ} \mathrm{C}$.

Como esta detallado en la subsección Análisis de contexto climático donde se identifican las temperaturas aceptables de CTMA para la ciudad de barranquilla al interior de un recinto, la temperatura media deseada para el análisis planteado es de $25.1{ }^{\circ} \mathrm{C}$ como temperatura optima, con máximos y mínimos de aceptación $2{ }^{\circ} \mathrm{C}$ superiores e inferiores a esta temperatura, esto establece temperaturas aceptables al interior de los recintos dentro de los 23.1 y $27.1^{\circ} \mathrm{C}$.

\section{AMBIENTE EXPERIMENTAL}

Ambiente experimental: el contexto de simulación definido sitúa el espacio en la ciudad de Barranquilla con fecha de simulación el 18 de abril, con cinco horas de análisis, con el objetivo de realizar un seguimiento detallado al comportamiento térmico del espacio de simulación a lo largo del día.

La simulación realiza de manera simultánea un grupo de cinco análisis dentro de los cuales encontramos evaluación de Temperatura operativa (to) y evaluación de Confort adaptativo (CTMA) que son los ejercicios determinados para el análisis de resultados (ANSI/ASHRAE, 2017).

Frente al análisis anterior se evalúan las envolventes con el siguiente panel de resultados:

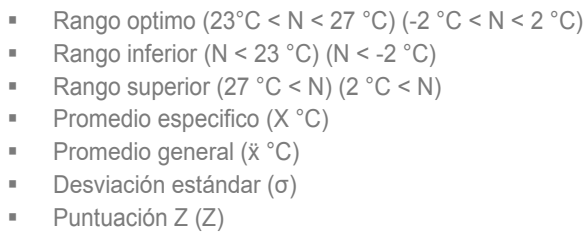

Grupos de tratamiento: la simulación cuenta con dos grupos de tratamiento, el primero denominado grupo de control, que maneja dos estados de análisis, un caso sin envolvente de protección o en estado actual del edificio con ventanería sin elementos de protección solar y un segundo estado con parasoles horizontales de $30 \mathrm{~cm}$ en voladizo espaciados verticalmente cada $30 \mathrm{~cm}$; el grupo experimental considera las cuatro EC definidas con anterioridad:
E01 RESPONSIVE SUN-SHADING SYSTEM (2014) Rodrigo Velasco, Frontis 3D, Bogotá, Colombia.

E02 NORDIC EMBASSIES IN BERLIN (1999) - Alfred Berger, Tiina Parkkinen, Berlin, Alemania

E03 ADAPTIVE SOLAR FACADE (ASF) (2016) - Zoltan Nagy and partners, Zurich, Suiza.

E04 FACHADA KIEFER TECHNIC SHOWROOM (2007) Giselbrecht + Partner ZT GmbH, Estiria, Austria.

Variables: dentro del análisis se consideran dos tipos de variables las variables dependientes y las variables independiente, en este caso, las variables dependientes corresponden al grupo de envolventes cinéticas y específicamente al desempeño que estas obtengan con el análisis de CTMA; las variables independientes están determinadas por la variaciones que estas puedan generar a la variable dependiente en el contexto de simulación, es por esto que los grados de movimiento de cada una de las envolventes serán considerados como las variables independientes, que están medidos cada $10^{\circ}$ de movimiento ya sea en el eje horizontal o en el eje vertical.

Selección de posiciones: la selección de la posición adecuada para el sistema de envolvente en este caso es regida directamente por la condición de Nodos en rango, el Promedio especifico y el Promedio general, es por esto que, la posición seleccionada es solo una, sin importar el número de posiciones que se puedan experimentar.

Finalmente, el modo en el que se presenta la información en cada uno de los siguientes subcapítulos correspondientes a cada caso evaluado con gráficas y una descripción general de lo sucedido en el día.

\section{EOX - ENVOLVENTE MUROS CORTINA, SISTEMA ACRISTALADO, ESTADO ACTUAL DE EDIFICACIÓN.}

El primer caso dentro del grupo de control es la simulación de envolvente en condiciones actuales del espacio de simulación, es importante recalcar que para esta simulación la ventaneria no cuenta con elementos de protección solar y tiene una especificación de vidrio laminado $4+4$ sin película de baja emisividad, esta especificación se utilizara además para las simulaciones siguientes; dentro de los tres análisis gráficos que se pueden observar en la Figura 5 a lo largo del día, las condiciones de CTMA dentro de un máximo de $2^{\circ} \mathrm{C}$ tienen un área menor a un tercio del área completa del espacio, 
donde predominan condiciones de CTMA superiores a los $3^{\circ} \mathrm{C}$ y con una temperatura promedio durante el día de 29.5 ${ }^{\circ} \mathrm{C}$, es decir $4.5^{\circ} \mathrm{C}$ sobre la temperatura máxima de CTMA adecuada.

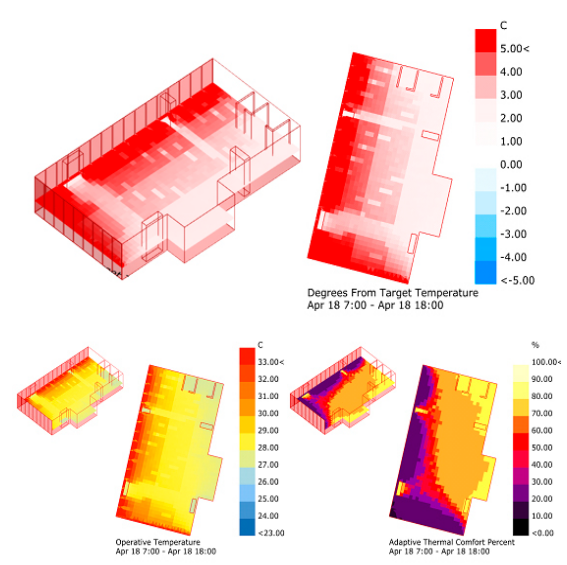

Figura 5: Plantas de análisis para E0X h: 7:00 am - 18:00 pm, simulación para CTMA, to y PCT.

EOY - ENVOLVENTE PARASOLES HORIZONTALES DE 30 CM CON ESPACIADO VERTICAL CADA 30 CM.
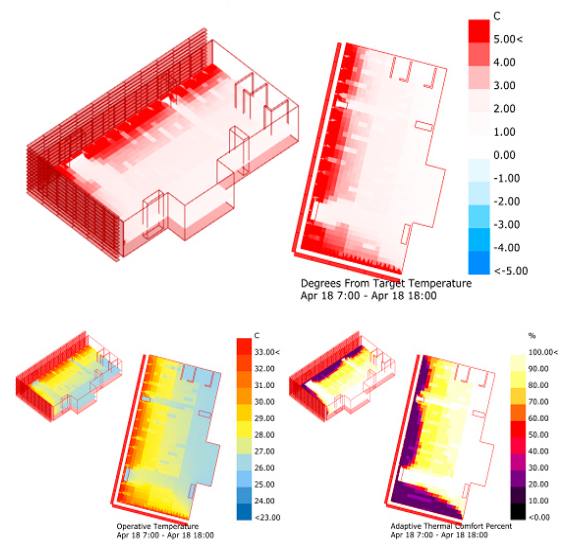

Figura 6: Plantas de análisis para E0Y h: 7:00 am - 18:00 pm, simulación para CTMA, to y PCT.

El segundo caso del grupo de control propone un estado de simulación con un sistema de envolvente compuesto por elementos fijos, son parasoles horizontales de $30 \mathrm{~cm}$ con separaciones verticales cada $30 \mathrm{~cm}$ que están caracterizados dentro del algoritmo de simulación como elementos de aluminio; lo que se puede observar en la Figura 6 con la visualización de los resultados de CTMA es un aumento importante de área dentro de los grados de aceptación de temperatura, más de la mitad del área se ocupa por el color correspondiente al máximo de aceptación de $2{ }^{\circ} \mathrm{C}$ y de $1{ }^{\circ} \mathrm{C}$, aunque no se alcanza a visualizar área de aceptación en temperatura optima con 0 ${ }^{\circ} \mathrm{C}$ el aumento de área con respecto a EOX es importante a lo largo del día, además la temperatura promedio del día es de $27.8^{\circ} \mathrm{C}$, es decir $2.8^{\circ} \mathrm{C}$ superior al máximo aceptado de CTMA.
E01 - RESPONSIVE SUN-SHADING SYSTEM (2014) RODRIGO VELASCO, BOGOTÁ, COLOMBIA.

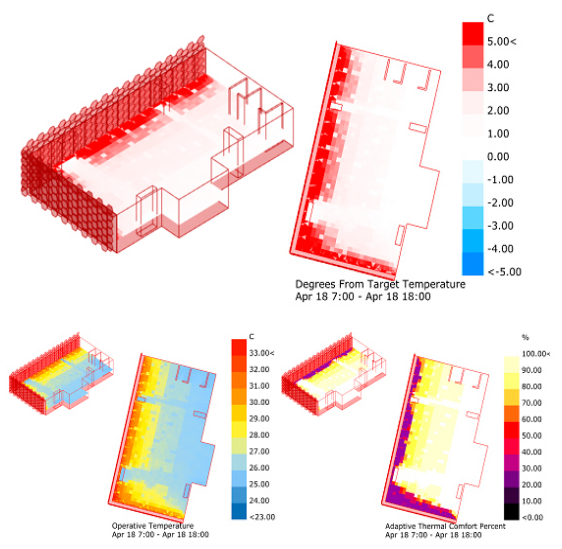

Figura 7: Plantas de análisis para E1 h: 7:00 am - 18:00 pm posición $10^{\circ}$, simulación para CTMA, to y PCT

El primer caso del grupo experimental es una EC desarrollado a nivel de prototipo compuesto por módulos hexagonales laminares rígidos, este sistema de envolvente cuenta con una característica de recolección de energía mediante células fotovoltaicas en uno de sus lados, sin embargo, la caracterización de material para la simulación es propuesta en aluminio y se replican los dos grados de libertad de movimiento. Como se puede observar en la Figura 7 el área en el gráfico de CTMA que corresponde a rangos de aceptación adecuados es superior a dos tercios del área total del espacio de simulación, este caso presenta un incremento importante con respecto a los dos casos de simulación del grupo de control, aún más importante en este caso la temperatura promedio del día es de $26.1^{\circ} \mathrm{C}$ tan solo $1{ }^{\circ} \mathrm{C}$ superior a la to optima y además dentro del rango de temperatura aceptable de CTMA.

\section{E02 - NORDIC EMBASSIES IN BERLIN (1999) - ALFRED B., TIINA P., BERLIN, ALEMANIA.}
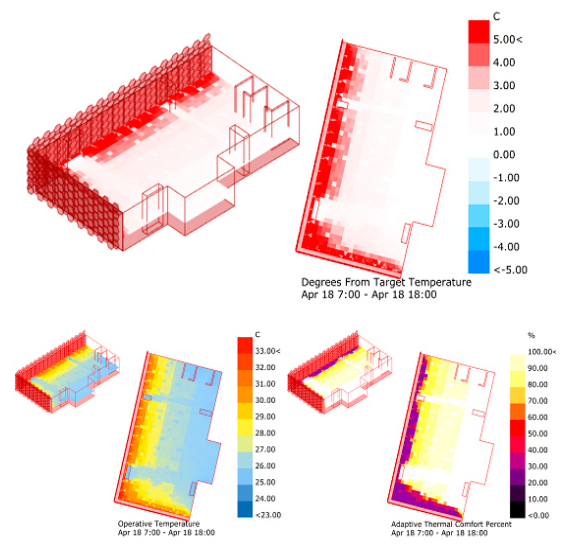

Figura 8: Plantas de análisis para E2 h: 7:00 am - 18:00 pm posición $10^{\circ}$, simulación para CTMA, to y PCT. 
El segundo caso del grupo experimental es una EC desarrollado como piel de un conjunto de 5 edificaciones independientes, el sistema está compuesto por unas persianas que cuentan con un grado de libertad en rotación y tienen un sentido de apertura descendente con un rango de rotación que va desde $0^{\circ}$ en estado de cierre hasta $90^{\circ}$ en estado de apertura; para la caracterización en el algoritmo de simulación se le dieron propiedades de aluminio por ser este el elemento principal del sistema. En la Figura 8 de igual manera que en el caso anterior de la E01 el área del gráfico de CTMA en rangos de aceptación supera los dos tercios del área total, de igual forma hay un aumento importante con respecto a los casos del grupo de control, además la EC presenta un promedio de to a lo largo del día de $26.6{ }^{\circ} \mathrm{C}$ que también es una temperatura que se encuentra dentro del rango de aceptación de CTMA menor a $27.1^{\circ} \mathrm{C}$.

\section{E03 - ADAPTIVE SOLAR FACADE (ASF) (2016) -} ZOLTAN NAGY AND PARTNERS, ZURICH, SUIZA.

Sistema compuesto por módulos con forma de rombo que tienen un funcionamiento similar al presentado por la E01 donde se tiene la función de recolección de energía por medio de una película fotovoltaica adherida a la cara principal del sistema, cuenta con dos grados de libertad de $90^{\circ}$ en sentido vertical y horizontal; los elementos en el algoritmo de simulación son caracterizados con aluminio como material principal pues en este caso las películas fotovoltaicas se adhieren a laminares metálicos. También en la Figura 9 de igual forma que en el segundo sistema de envolvente se llega a un promedio de to a lo largo del día de $26.6^{\circ} \mathrm{C}$ y además como sucede en los demás casos experimentales el área dentro de rangos de confort es similar en una proporción de dos tercios sobre el área total.
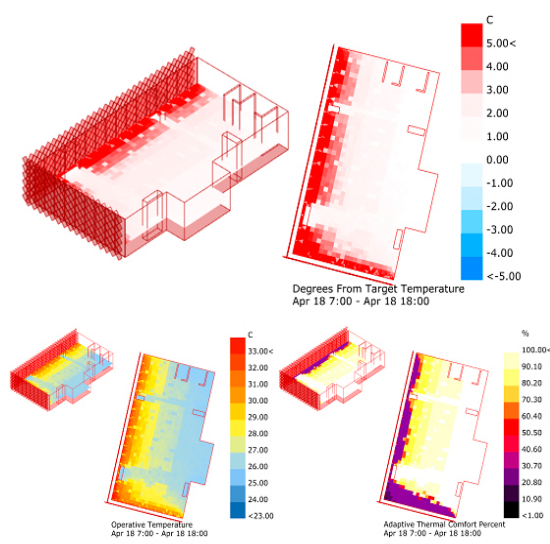

Figura 9: Plantas de análisis para E3 h: 7:00 am - 18:00 pm posición $10^{\circ}$, simulación para CTMA, to y PCT.

\section{E04 - FACHADA KIEFER TECHNIC SHOWROOM (2007) - GISELBRECHT + PARTNER ZT GMBH, ESTIRIA, AUSTRIA.}

El cuarto caso es un sistema de persiana doble, compuesta por módulos articulados, el sistema se pliega o despliega con ayuda de rieles laterales; estos módulos se componen de láminas micro perforadas metálicas blancas, es por esto que la caracterización en este caso para el algoritmo es de aluminio, además la graduación en el sistema propone quince posiciones de despliegue donde -1.5 es la condición de cierre y - 0.1 es la condición de apertura. En este caso en la Figura 10 se observa un mínimo aumento de área en to dentro de rangos de aceptación, sin embargo se debe resaltar que este sistema de envolvente es considerablemente menos permeable a la luz que los casos anteriores dentro del grupo de experimentación, debido al tamaño de los módulos, la temperatura promedio a lo largo del día alcanza los $26.5^{\circ} \mathrm{C}$ que igualmente se encuentra dentro de rangos de aceptación.

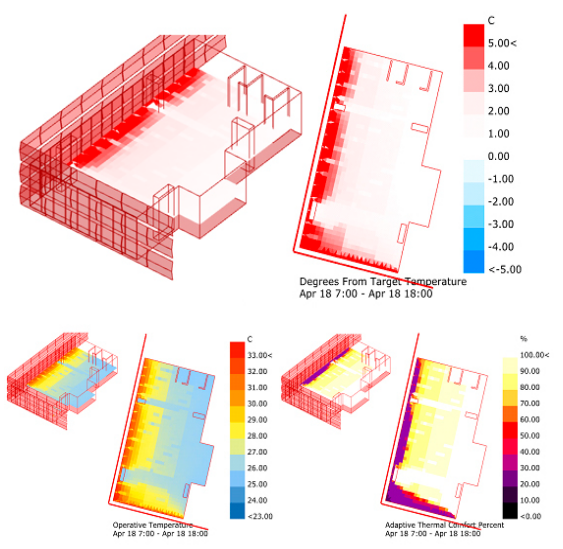

Figura 10: Plantas de análisis para E4 h: 7:00 am - 18:00 pm posición -1.3, simulación para CTMA, to y PCT.

\section{ANÁLISIS DE RESULTADOS}

El análisis deriva de un ejercicio de simulación caracterizado por contener un ambiente experimental determinado, dos grupos de tratamiento y un conjunto de variables descritas con mayor detalle en el apartado anterior; producto de esta simulación se recolectan desde un panel de resultados nueve datos de respuesta, a partir de una superficie con 1696 nodos de lectura repartidos en la totalidad del área del espacio de simulación. Finalmente se extraen del conjunto de once mil ochocientos cincuenta (11850) simulaciones un numero de tres mil trescientos treinta (3330) datos de respuesta.

Desde una primera observación se pueden distinguir un grupo de datos que no genera ningún tipo de información relevante al análisis, este es la condición de nodos de lectura por debajo de rangos de to aceptable $\left(\mathrm{N}<23^{\circ} \mathrm{C}\right)$ puesto que para todos los casos en cada una de las posiciones la respuesta es cero (0) lo cual permite definir una única conclusión para este dato y es que las condiciones de temperatura permanentemente se mantienen por encima de los $23.1^{\circ} \mathrm{C}$. También se puede observar dentro de las tablas de datos que las diferencias de temperatura entre estados de cierre y apertura en las $E C$ en las primeras tres horas de análisis (7:00 am, 10:00 am, 12:00 pm) es inferior a $1^{\circ} \mathrm{C}$ sin embargo en las últimas dos horas de análisis (3:00 pm, 5:00 pm) estas diferencias se amplían hasta márgenes de casi $2.5^{\circ} \mathrm{C}$.

Determinar las posiciones de EC para cada caso es uno de los últimos pasos de la investigación con la intención de plantear un balance y comparativo de las curvas de to a partir de las posiciones de movimiento definidas para cada hora del día estudiado, el método para definir estas 
posiciones hace uso de la simulación descrita anteriormente, bajo estándares de CTMA (ANSI/ASHRAE, 2017), se arrojó un grupo de resultados en términos de to para cada posición opcional de $E C$, esto se ve reflejado en las gráficas de curva de to de la Figura 11.

\section{ANÁLISIS FINAL}

De acuerdo al análisis individual del comportamiento de las $E C$ del grupo experimental enfocado en los resultados de Rango optimo y Promedio general de to se estableció no solo la relación de los datos sustraídos en el panel de resultados, también se debe destacar como lo muestra cada una de las gráficas que la relación entre el las posición de cierre y apertura de las EC y su comportamiento térmico está directamente relacionado, en todos los casos analizados se puede observar en las gráficas que en la posición de cierre se concentran los mejores resultados de to que conforme van avanzando las horas del día van adquiriendo mayor margen de incremento en los valores de resultado.

Por lo anterior en la Figura 12 se concentran los resultados de temperaturas definidas por las posiciones seleccionada en el apartado anterior para cada una de las EC del grupo experimental junto con las temperaturas resultantes en los casos de los sistemas de envolvente del grupo de control (Tabla 2), estos resultados se visualizan en el eje x para las horas del día que fueron simuladas, esto con la intención de generar una curva de comportamiento diario. Esta es una de las gráficas más informativas generadas dentro del marco del estudio realizado arrojando comportamiento térmico, picos de temperatura, diferencias en sistemas de envolvente, así como las similitudes de las mismas, y esto también trasladado a consumo de energía

E01 RESPONSIVE SUN-SHADING SYSTEM (2014)

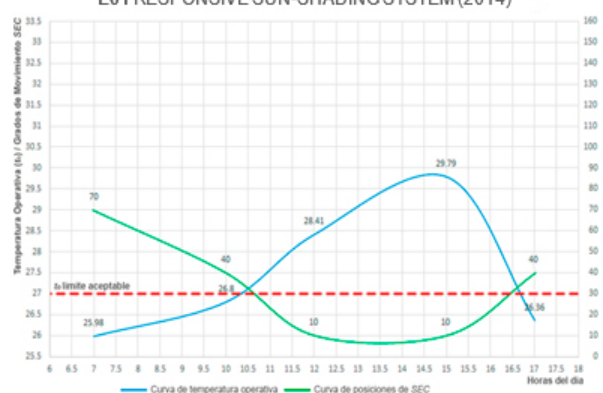

E03 ADAPTIVE SOLAR FACADE (ASF) (2016)

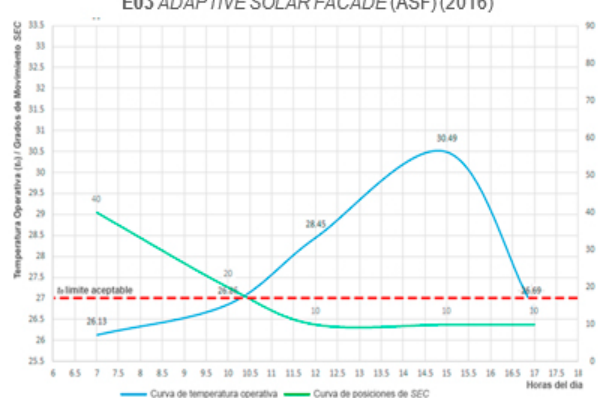

Figura 11: Grafica de curva de to y curva de posiciones de movimiento de EC E01, E02, E03, E04. por el número de horas de necesidad de uso de sistemas mecánicos de enfriamiento

Tabla 2: Valores de to a lo largo del día en el Grupo de control y Grupo Experimental (SEC).

\begin{tabular}{c|cc|cccc}
\hline & & E0Y & E1 & E2 & E3 & E4 \\
\hline $7: 00$ & 26.34 & 26.06 & 25.98 & 26.10 & 26.13 & 26.17 \\
$10: 00$ & 27.82 & 27.19 & 26.80 & 26.70 & 26.86 & 26.76 \\
$12: 00$ & 30.08 & 29.41 & 28.41 & 28.39 & 28.45 & 28.46 \\
$15: 00$ & 33.14 & 30.54 & 29.79 & 30.45 & 30.49 & 29.85 \\
$17: 00$ & 29.88 & 27.76 & 26.36 & 26.65 & 26.69 & 27.13 \\
\hline
\end{tabular}

Las curvas de comportamiento de to que se observan en la Figura 12 agrupan los resultados para los casos analizados en el grupo de control y en el grupo experimental; el primer caso del grupo de control es E0X, esta curva de resultados muestra un comportamiento ascendente prolongado a lo largo del día que se separa visiblemente de las demás curvas haciendo notoria la necesidad de un sistema de protección solar ya sea estático o dinámico, de esta curva de resultados se destaca que sale de los límites de temperatura aceptable aproximadamente a las 8:30 am, limite al que no vuelve a lo largo del día en ningún momento. El segundo caso del grupo de control es EOY un sistema de envolvente de elementos estáticos, la curva de resultados para este caso describe un mejor comportamiento, sin embargo, no demuestra el mejor desempeño, en este caso se puede concluir que la curva sale del límite de temperatura aceptable aproximadamente a las 9:30 am y no vuelve a entrar en ningún momento del día.

El análisis del grupo experimental de EC tiene un margen de diferencia importante solo visualizado en la Figura 12 a
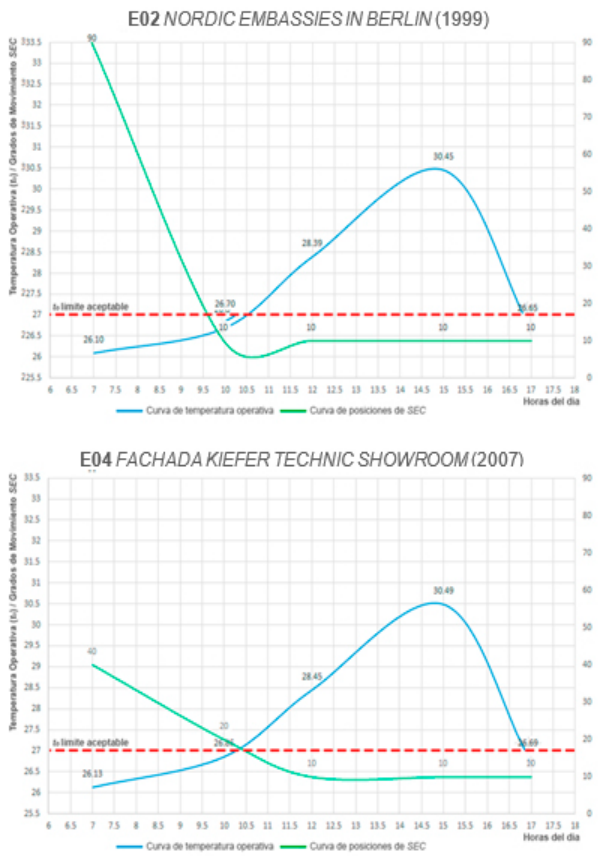


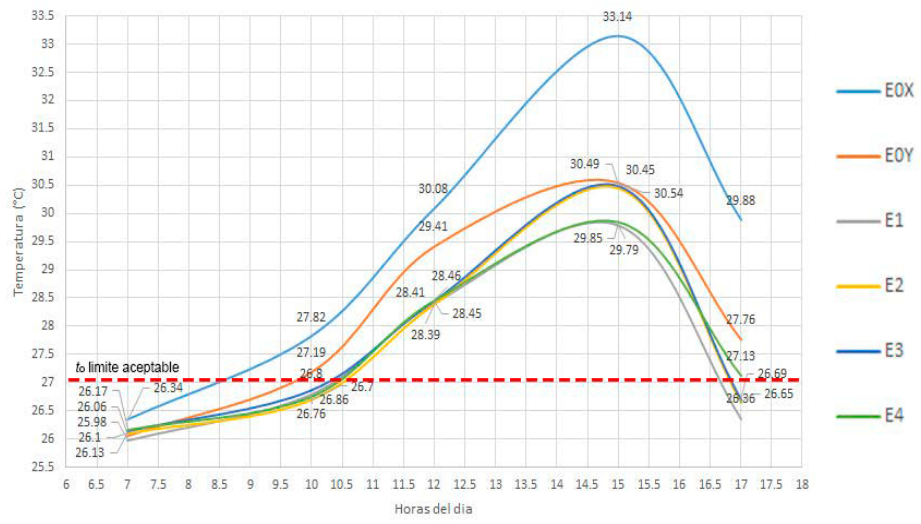

Figura 12: Grafica de curvas de to a lo largo del día de temperaturas mínimas en cada EC.

partir de las 12:00 pm pues antes de eso los cuatro casos mantienen un nivel de semejanza en sus resultados que inclusive a las 3:00 pm solo tiene un margen de diferencia de $0.6^{\circ} \mathrm{C}$, en el caso de $\mathrm{E} 2$ y E3 que son las $E C$ con mayor nivel de semejanza, en ambos casos se debe destacar que salen del límite de temperatura aceptable cercanos a las 10:30 am y regresan cercanos a las 4:45 pm. Los dos casos restantes E1 y E4 también tienen un comportamiento similar en resultados que se diferencia solo al final de la tarde, en estos dos casos para E1 se puede considerar que la curva de temperatura sale del límite aceptable a las 10:30 am para volver a las 4:45 pm y en el caso de E4 saliendo también a las 10:30 se puede determinar que vuelve al límite de temperatura media hora después a las 5:15 pm.

\section{CONCLUSIONES}

Las EC estudiadas confirman la necesidad de incrementar la investigación en el campo de desarrollo cinéticos en envolventes, al llegar a la conclusión de que no existe un perfil estricto que además sea comparativamente superior frente a otros, por el contrario este se construye con la unión de diferentes rasgos, un perfil mixto que evoca diferentes líneas de diseño, pero también con un grupo de cualidades especificas centradas en la baja complejidad y buen desempeño con la premura de optimización de casos existentes. Se debe resaltar que los sistemas celulados y de disposición horizontal se mostraron de forma más apta frente a las condiciones estudiadas, asimismo los usos de configuraciones de movimiento sencillas de rotación en un grado de libertad se determinaron como las más utilizadas, soportadas en la idea de lograr el menor costo de sistema debido a que mecanismos con dos o más grado de movimiento pueden aumentar el costo de forma injustificada, algo que también se observa en las tablas de resultados por lo que en los dos casos de envolventes con dos grados de libertad no hay un aumento relevante en el desempeño al estudiar el segundo grado de libertad de movimiento, puesto que el movimiento en un solo eje puede generar resultados representativos de los dos ejes, sin embargo es un tema que por sí mismo puede constituir un proyecto de investigación, también se debe destacar que los dos casos de dos grados de libertad no tuvieron un comportamiento significativamente similar en la última etapa de estudios, por el contrario cada uno hace parte de los dos trayectos representativos del grupo experimental, por lo tanto esta diferencia puede presentarse por el cambio de geometría o proporciones del sistema. Con respecto al ahorro energético lograr un diferencial de hasta 4 horas de uso de sistemas de climatización, aunque no alcanza un ahorro total si representa más de una tercera parte de periodo de uso en un día típico laboral en la ciudad de Barranquilla sumado a la disminución de $4{ }^{\circ} \mathrm{C}$ en el pico de temperatura típico en el día, lo que por sí mismo significa un antecedente positivo y favorable de investigación que con el avance en optimizaciones puede significar hasta ahorros de más de la mitad de los periodos de tiempo en uso, lo que significa una disminución de consumo relevante.

Para finalizar, el trabajo realizado se soporta en la idea de fortalecer la investigación, el desarrollo y construcción de alternativas que tengan el objetivo de mejorar no solo condiciones estéticas y tecnológicas en las edificaciones, también condiciones de satisfacción ambiental para el usuario y reducir los impactos al medio en el que se ubican los proyectos construidos.

\section{REFERENCIAS}

ANSI/ASHRAE. (2017). Standard 55: 2017, Thermal Environmental Conditions for Human Occupancy.

Japan Refrigeration and Air Conditioning Industry Association. (2019). World Air Conditioner Demand by Region.

Kottek, M., Grieser, J., Beck, C., Rudolf, B., \& Rubel, F. (2006). World Map of the Köppen-Geiger Climate Classification Updated. Meteorologische Zeitschrift, 15, 259-263. https://doi.org/10.1127/0941-2948/2006/0130

López, M., Rubio, R., Martín, S., \& Ben Croxford. (2017). How plants inspire façades. From plants to architecture: Biomimetic principles for the development of adaptive architectural envelopes. Renewable and Sustainable Energy Reviews, 67, 692-703. https://doi.org/https://doi.org/10.1016/j.rser.2016.09.018

Mackey, C., \& Roudsari, M. S. (2017). The Tool(s) Versus The Toolkit. In In Proceedings of the Design Modeling Symposium

Mostapha Sadeghipour Roudsari. (2013). Ladybug Tools. https://www.ladybug.tools/index.html

Robert McNeel \& Associates. (2013). Rhinoceros 3D. Miami, USA. https://www.rhino3d.com/

Velasco, R., Brakke, A., \& Chavarro, D. (2015). Dynamic Façades and Computation: Towards an Inclusive Categorization of High Performance Kinetic Façade Systems. https://doi.org/10.1007/978-3-662-47386-3_10 